\title{
Liste commentée des Cylapinae d'Argentine et du Chili avec la description d'un nouveau genre et d'une nouvelle espèce (Hemiptera, Heteroptera, Miridae)
}

\author{
par Diego Leonardo CARPINTERO* \& Frédéric CHÉROT** \\ * División Entomología, Museo Argentino de Ciencias Naturales "Bernardino Rivadavia", Av. Ángel Gallardo 470 \\ (1405), Ciudad Autónoma de Buenos Aires, Argentina <dcarpint@macn.gov.ar> \\ ** Service Public de Wallonie, DGO3, DEMNA, avenue du Maréchal Juin 23, BE - 5030 Gembloux, Belgique \\ $<$ frederic.cherot@spw.wallonie.be> \\ urn:1sid:zoobank.org:pub:CDE2765A-652F-4243-BC02-3A9DB61B6262
}

Résumé. - Une liste actualisée des Cylapinae d'Argentine et du Chili est fournie. Fulvius vicosensis Ferreira \& Henry, 2002, est mentionné pour la première fois d'Argentine. Xenocylapoides gorczycai n. gen., n. sp., est décrit comme nouveau genre et nouvelle espèce de Curicó au Chili sur la base de l'holotype mâle et de cinq paratypes des deux sexes. Les structures génitales mâles et l'habitus des deux sexes sont figurés.

Abstract. - Annotated list of Cylapinae from Argentina and Chile with description of a new genus and species (Hemiptera, Heteroptera, Miridae). An updated list of Cylapinae from Argentina and Chile is provided. Fulvius vicosensis Ferreira \& Henry, 2002, is recorded for the first time from Argentina. Xenocylapoides gorczycai n. gen., n. sp., is described as new genus and species from Curicó, Chile, based on the male holotype and five paratypes of both sexes. The male genital structures and the habitus of both sexes are figured.

Keywords. - Cylapini, Fulviini, taxonomy, new genus, new species, Chile, Argentina, new record, Fulvius vicosensis, species list.

Les Cylapinae de la partie méridionale du continent sud-américain restent peu étudiés. CARVAlHo (1957) ne mentionne qu'une seule espèce de la sous-famille pour l'Argentine dans son catalogue des Miridae du Monde, à savoir Fulvius bisbistillatus (Stål, 1860), espèce qui est peutêtre aussi présente au Chili. Dans leur catalogue des Miridae d'Argentine, CARPINTERo \& CARVALHO (1993) ne citent que cinq espèces, toutes des Fulviini, dont trois Fulvius Stål, 1862. Huit autres espèces seront ultérieurement mentionnées dans la littérature pour l'Argentine (CARPINTERO, 1998, CARPintero \& CARvalho, 1993, CARPintero et al., 2006, Dellapé et al., 2010, Wolski \& Henry, 2012), une neuvième (Fulvius vicosensis Ferreira \& Henry, 2002) l'étant pour la première fois dans le présent travail. En revanche, aucune espèce additionnelle n'est citée pour le Chili voisin, dont la faune des Miridae est encore moins connue et apparemment moins riche.

Nous fournissons ci-dessous une première liste commentée des Cylapinae actuellement connus d'Argentine et du Chili, avec mention d'observations nouvelles, et une description d'un petit Fulviini chilien que nous ne pouvons attribuer à un genre et à une espèce déjà décrits, d'où la création d'un nouveau genre. Ceci porte à deux le nombre de Cylapinae connus du Chili, pour 13 d'Argentine, des nombres très certainement largement sous-estimés.

Le matériel étudié est préservé dans les collections suivantes : CC, collection Diego Carpintero, Quilmes, Argentine; ISNB, Institut royal des Sciences naturelles de Belgique, Bruxelles; MACN, Museo Argentino de Ciencias Naturales "Bernardino Rivadavia", Buenos-Aires, Argentine; MHNC, Museo de Historia Natural de Chile, Santigo, Chili.

Sous-famille des Cylapinae Kirkaldy, 1905

Tribu des Cylapini Kirkaldy, 1905

Cylapocoris sulinus Carvalho \& Gomes, 1971. Argentine : Misiones (Dellapé et al., 2010). 
Valdasus bolivianus Carvalho, 1989. Argentine : Misiones (CARPINTERo, 1998, comme un Cylapus non identifié; CARPINTERO et al., 2006).

Tribu des Fulviini Uhler, 1896

Comefulvius chingonus Carvalho \& Carpintero, 1985. - Argentine : Córdoba (CARValHo \& CARPINTERo, 1985).

Fulvius amapaensis Carvalho \& Costa, 1994. - ARgentine : Misiones (CARPINTERo et al., 2006).

Fulvius bisbistillatus (Stål, 1860). - ARGENTINE : Buenos Aires (NANNI et al., 2011), Chaco (CARPINTERO \& Carvalho, 1993 ; Melo et al., 2011), Corrientes (Melo et al., 2004), Misiones, Salta (CARPintero \& CARvalho, 1993).

Nouvelles observations. Argentine : Formosa : 1 क, E[stanci]a La Marcela, 35 km E[ste] El Colorado, (luz), VII.2003, J. Williams (CC); Tucumán : 1 đે, El Cadillal, III.2003, Cicchino (CC); Santiago del Estero : 1 ô, 1 \%, Añatuya, XI.1998, UV Light, Carpintero D. J. (CC). CHILI : Catín (Araucania) (CARVAlho \& Afonso, 1977, comme F. bistillatus, une graphie incorrecte), Tarapaca (GORCZYCA, 2006).

On notera que Carvalho (1957 : 15-16) ne cite pas explicitement F. bisbistillatus du Chili, se contentant de considérer l'espèce comme néotropicale et de mentionner le Brésil et le Panama. En revanche, Carvalho \& Afonso (1977) et Gorczyca (2006: 44) citent, eux, clairement le Chili. CARvalho \& Afonso (1977) se basent sur un nombre non précisé de spécimens de l'Académie des Sciences de Californie, dont ils n'indiquent pas le sexe et qu'ils ne considèrent apparemment pas comme nouveaux pour le Chili (pas d'astérisque). GorczyCA (2006) reprend la donnée de CarvalHo \& AFONSO (1977) dans sa distribution générale et mentionne en outre, dans une liste de données originales accompagnant le catalogue, un spécimen de la ville d'Azapa, province de Tarapaca, préservé à Budapest. Il s'agit malheureusement d'une femelle. Or, les Fulvius du Nouveau Monde sont nombreux et souvent assez similaires d'habitus, le travail de synthèse de CARVALHO \& COSTA (1994) se base largement sur les génitalias des mâles et, à l'époque, les génitalias femelles de $F$. bisbistillatus n'étaient pas décrits. Faute d'une analyse de ces génitalias, un doute subsiste donc quant à l'identification spécifique. Prado (2008), dans son catalogue des Hétéroptères du Chili, doute d'ailleurs de la présence de $F$. bisbistillatus dans son pays et il y a effectivement lieu de s'interroger dans la mesure où il n'est pas certain que s'y trouve un environnement qui lui soit favorable. En conséquence, la présence de F. bisbistillatus au Chili reste à prouver, par une nouvelle analyse des spécimens de Budapest et de San Francisco, et l'étude de matériel additionnel. En revanche, la présence du genre Fulvius au Chili paraît assurée.

Fulvius bolivianus Carvalho \& Costa, 1994. - Argentine : Jujuy (CARPINTERo et al., 2006).

Fulvius breddini Reuter, 1902. - Argentine : Misiones (CARPintero \& CARvalho, 1993).

Nouvelles observations : Argentine: Corrientes : $1 \hat{\jmath}$, S[an]ta Tecla, (Departamento Ituzaingó), III.1994, CDC (trap), Carpintero (CC); 1 +, idem, VII.1999 (CC).

Fulvius chaquenus Carvalho \& Costa, 1994. - Argentine : Buenos Aires (CARPIntero, 2009), Corrientes, Entre Ríos [CARPINTERo \& CARVALHo, 1993, identifié comme F. quadristillatus (Stål, 1860)], Misiones (CARvalho \& Costa, 1994).

Fulvius itabiritensis Carvalho \& Costa, 1994. - ARgEnTINE : Misiones (CARPINTERo et al., 2006).

Fulvius quadristillatus (Stål, 1860). - Argentine : Chaco, Misiones, Salta, Tucumán (CARPINTERo \& Carvalho, 1993).

Fulvius vicosensis Ferreira \& Henry, 2002. - Nouvelle espèce pour la faune argentine. ARGENTINE : Chaco : 1 ภ, 1 + , R[eserva] Loro Hablador, D[epartamen]to. G[eneral] Güemes, Luz, XII.2006, Gerardo Marti (CC).

Peritropis amphicryta Wolski \& Henry, 2012. - Argentine : Misiones (Wolski \& Henry, 2012).

Peritropis carpinteroi Wolski \& Henry, 2012. - Argentine : Misiones (CARPINTERo \& CARvalho, 1993, identifié comme $P$. saldaeformis Uhler, 1891 ; WOLSKI \& HENRY, 2012).

Peritropis guarani Wolski \& Henry, 2012. - Argentine : Buenos Aires (Wolski \& Henry, 2012), Chaco (CARPINTERo et al., 2006, identifié comme P. unicolor Carvalho \& Rosas, 1965 ; WolSKI \& HenRY, 2012).

Xenocylapoides gorczycai n. gen., n. sp. - CHILI : Curicó. 


\section{Xenocylapoides n. gen.}

urn:1sid:zoobank.org:act:ACE40297-994A-4B3F-BAF9-34B593C95434

Espèce-type : Xenocylapoides gorczycai $\mathrm{n}$. sp.

Diagnose. - Petit Cylapinae Fulviini; tête quasi horizontale; premier article antennaire allongé, incurvé, dépassant l'extrémité antérieure de la tête; labium fin et allongé, atteignant le milieu de l'abdomen; premier article du rostre atteignant l'extrémité postérieure de la tête; collier pronotal bien visible; callosités pronotales présentes, grandes, arrondies, non tuberculeuses; profémurs larges juste après la base; cunéus nettement délimité chez le mâle — néanmoins sans qu'il y ait de véritable fracture cunéale — mais absent chez la femelle submacroptère; surface dorsale lisse (dépourvue de rangées de points ou de toutes autres formes de ponctuation) porteuse d'une pilosité blanche extrêmement courte, couchée ou semi-dressée, à peine visible.

Derivatio nominis. - Nommé par référence aux deux genres semblant les plus voisins, Xenocylapus Bergroth, 1922, et Xenocylapidius Gorczyca, 1997.

Discussion. - Xenocylapoides n. gen. peut être classé dans la tribu Fulviini : par sa tête quasi horizontale, longue et pointue, au clypéus distinctement courbé, dont l'apex est ventral par rapport à la base (c'est-à-dire à la zone bordant le front); par ses callosités pronotales larges, confluentes, occupant plus du tiers de la surface pronotale. La présence d'un collier pronotal bien visible dans les deux sexes et l'habitus allongé permettent aisément de séparer le nouveau genre de Peritropis Uhler, 1891. Dès les premiers couplets, la clé des Cylapinae néotropicaux de Carvalho \& Ferreira (1994) est prise en défaut, le mâle de Xenocylapoides possédant un cunéus nettement délimité et donc distinct au sens de ces auteurs, contrairement à la femelle'. Si le cunéus du mâle est distinct et délimité de la corie, on notera cependant que c'est par un fin sillon blanc et non par une fracture cunéale au sens classique, les parties latéroexternes de la corie et du cunéus ne semblant pas complétement séparées.

L'habitus de la femelle de Brachyfulvius Carvalho, 1955, est plus nettement coléoptéroïde que celui de la femelle de Xenocylapoides n. gen. La division hémélytrale en clavus et corie n'est plus du tout visible chez le premier, alors qu'elle reste discernable chez le second. En outre, les callosités pronotales se terminent par deux protubérances coniques chez Brachyfulvius, protubérances absentes chez Xenocylapoides. La quasi-absence de pilosité hémélytrale conduit à séparer le nouveau genre d'Incafulvius Carvalho, 1976, bien caractéristique par sa pilosité dorsale éparse, raide, dressée, relativement allongée (CARVALHO, 1976). Les deux genres se distinguent en outre par la structure de leurs callosités pronotales. L'article I des antennes dépassant nettement l'apex de la tête et les callosités pronotales proéminentes séparent Xenocylapoides des genres Comefulvius Carvalho \& Carpintero, 1985, et Peritropoides Carvalho, 1955, le premier de ces genres étant en outre aisément reconnu par les lignes légèrement incurvées de larges points qui parcourent ses hémélytres.

Xenocylapoides n. gen. ne se rapproche dès lors, dans la faune néotropicale, que des genres Xenocylapus Bergroth, 1922, et Fulvius Stål, 1862. De Fulvius, le nouveau genre se sépare aisément par l'habitus globalement moins allongé, par la structure du cunéus — en particulier chez les femelles — et pour autant que l'on puisse en juger par les profémurs.

\footnotetext{
${ }^{1}$ On notera que cette clé devrait être revue à d'autres niveaux. Aucun critère n'est fourni pour séparer les tribus Cylapini et Fulviini, qui sont simplement incluses à la suite l'une de l'autre. Incafulvius Carvalho, 1976, dont le cunéus est noté p. 329 comme indistinct, est néanmoins classé sous le deuxième élément du couplet 2, p. 328, (renvoyant au couplet 4), deuxième élément dont le seul critère est "hémélytre avec cunéus distinct", ce qui est manifestement contradictoire. Valdasoides Carvalho, 1989, dont les pattes n'ont jamais été ni décrites, ni figurées, est lui classé avec Valdasus Stål, 1860, et Trichocylapus Poppius, 1909, sous le premier élément du couplet 6, p. 330, couplet fondé sur la longueur relative de la pilosité des métatibias.
} 


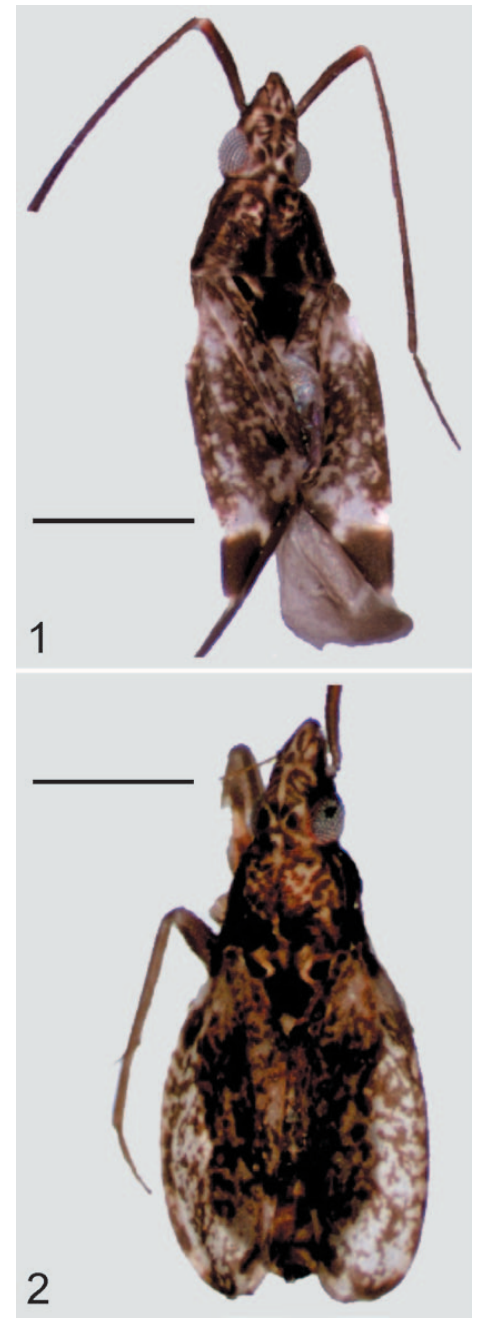

Fig 1-2. - Xenocylapoides gorczycai n. gen., n. sp., habitus en vue dorsale. -1 , Mâle paratype de l'ISNB. - 2, Femelle paratype de l’ISNB. Échelles : $1 \mathrm{~mm}$.
L'ensemble de ces caractères le rapproche nettement de Xenocylapus. Cependant, chez Xenocylapus, le cunéus est indistinct dans les deux sexes et le pédoncule antennaire est situé juste contre les yeux et pas au milieu de la tête. Xenocylapidius Gorczyca, 1997, de Nouvelle-Calédonie, présente un cunéus distinct chez la femelle (GoRCZYCA, 1997). Quant à Rhinofulvius Reuter, 1902, décrit d'Aden sur la base d'une seule femelle, ses hémélytres (qui portent quatre larges taches claires aux angles contrairement à ceux de Xenocylapoides $\mathrm{n}$. gen.) ne sont pas nettement séparés en clavus et corie, même si une délimitation s'y devine (GorCZYCA, 2000). Ils sont en outre nettement plus courts que l'abdomen, laissant libre la majorité des segments abdominaux en vue dorsale, alors qu'ils couvrent pratiquement tout l'abdomen chez Xenocylapoides n. gen. (tant mâle que femelle). Enfin, la membrane y est totalement absente, alors qu'elle est réduite à une fine bande formant un arc de cercle chez la femelle de Xenocylapoides n. gen., le bord postérieur des hémélytres y étant aussi nettement plus incurvé que chez Rhinofluvius, où, quoiqu'oblique, il est quasi droit.

Les genres Comefulvius, Incafulvius et Xenocylapus possèdent tous les trois un labium fin et allongé, un premier article rostral très court n'atteignant pas le milieu de la gula, et un deuxième article rostral nettement plus long que les articles trois et quatre. En outre, ces trois genres sont dépourvus d'aire d'évaporation métathoracique, l'ensemble de ces états de caractères semblant les grouper au sein des Fulviini du Nouveau Monde (CHÉRot et al., soumis). Xenocylapoides n. gen., quoiqu'ayant un labium allongé, atteignant le milieu de l'abdomen, se sépare par un premier article rostral plus long, atteignant l'extrémité postérieure de la tête en vue ventrale, un deuxième article rostral proportionnellement plus court et des aires d'évaporation métathoraciques bien présentes.

Xenocylapoides gorczycai $\mathrm{n} . \mathrm{sp}$.

urn:1sid:zoobank.org:act:77871B0B-1F44-4A0B-94D1-2E3560BD7A98

Holotype : `, Chili : Curicó, El Relvo, 20 km E Potero Grande (35¹1'13”S - 7056’07”W), 3.II.2004, J. E. Barriga leg. (collecté par "fogging" sur Nothofagus dombeyi) (MHNC).

PARATYPES : 2 o et 3 q, idem holotype ( 1 q MHNC, 1 ô et $1 q$ MACN, 1 ô et 1 q ISNB).

Description. - Mensurations en mm (paratypes de l'ISNB, respectivement $\hat{\delta}-\rho$ ). Longueur totale en vue dorsale (de l'extrémité antérieure du tylus à l'apex des hémélytres) : 4,10-3,75; largeur totale (prise au milieu des hémélytres) : 1,25-1,75; largeur des yeux : 0,15-0,15; largeur du vertex : $0,33-0,80$; longueur des articles antennaires : I : 1,30-0,73 ; II : 1,90-[article manquant]; III : 0,45-[article manquant]; IV : 0,23-[article manquant]; longueur du pronotum (collier inclus) : 0,63-0,63; largeur du pronotum (entre les angles huméraux) : 1,10-1,03; longueur 
du scutellum (mésoscutum exclu) : 0,38-0,38; largeur du scutellum : 0,43-0,5; longueur du cunéus : 0,63 -[non mesurable]; largeur du cunéus : 0,4-[non mesurable].

Mâle (fig. 1). Tête. Clypéus large, subhorizontal sauf l'apex légèrement incurvé, rouge-brun taché de jaune. Joues grandes, allongées, subtriangulaires, brunes antérieurement, dorsalement et ventralement,

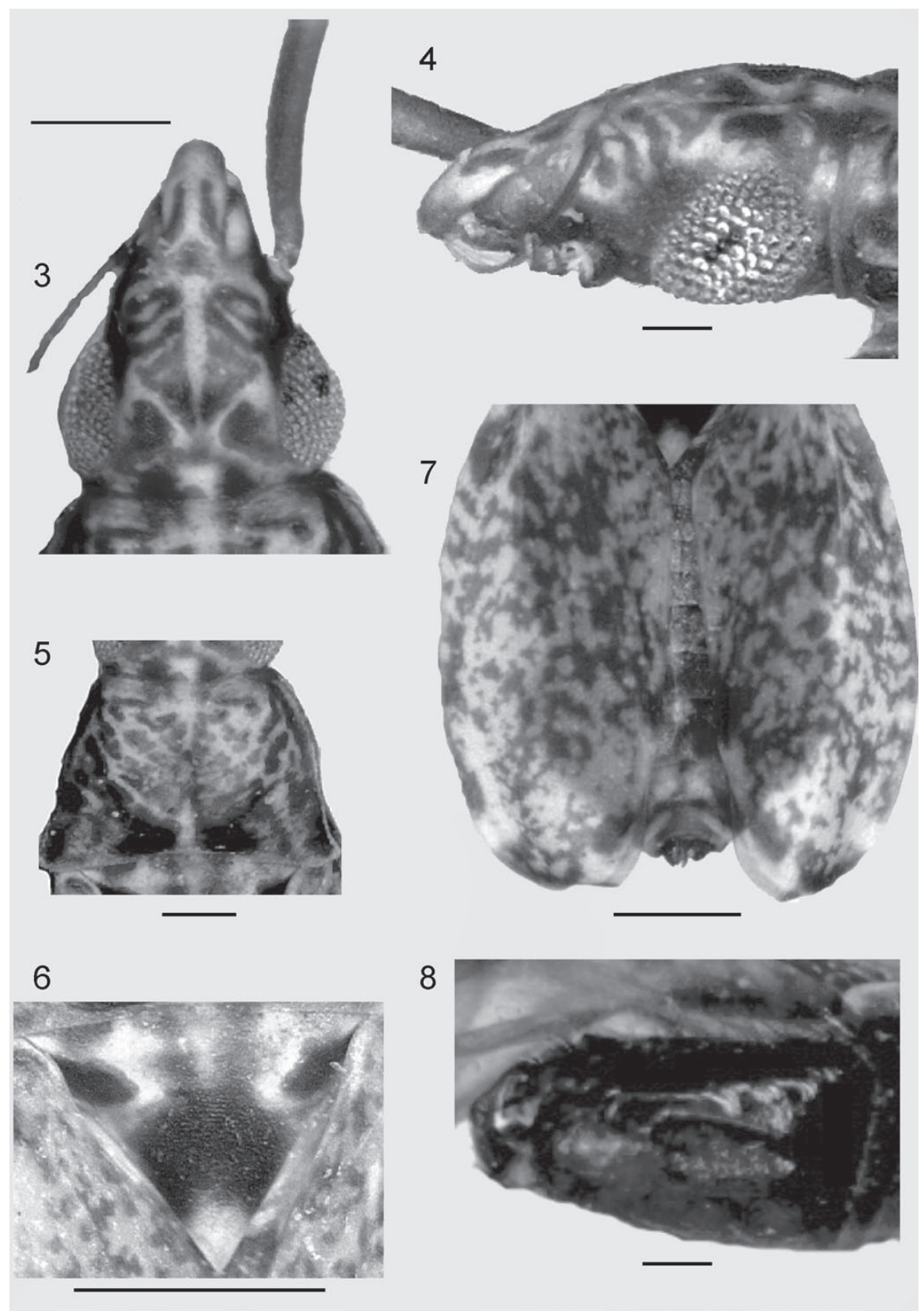

Fig. 3-8. - Xenocylapoides gorczycai n. gen., n. sp., détails de l'habitus. - 3-7, Femelle paratype de l'ISNB : 3, tête en vue dorsale (échelle : $0,5 \mathrm{~mm}$ ) 4 , tête en vue latérale (échelle : $0,1 \mathrm{~mm}$ ) 5 , pronotum en vue dorsale (échelle : 0,5 $\mathrm{mm}) ; 6$, scutellum en vue dorsale (échelle : $0,5 \mathrm{~mm}$ ) $;$, hémélytres (échelle : $0,5 \mathrm{~mm}$ ). -8 , Mâle paratype de l'ISNB, pygophore en vue latérale (échelle : $0,1 \mathrm{~mm}$ ). 
jaunes au milieu. Brides étroites, allongées, rouge-brun avec une bande jaune au milieu. Yeux argentés, apparemment glabres, les ommatidies grises. Fossettes antennaires nettement séparées des yeux, brunes, prenant naissance au bord inférieur des joues. Premier article antennaire s'épaississant très légèrement de la base à l'apex, faiblement incurvé, rouge-brun avec un fin anneau flave apical et portant de courtes soies blanches semi-dressées. Deuxième article antennaire brun, s'épaississant et s'assombrissant légèrement de la base à l'apex, couvert d'une pilosité semi-dressée de longueur similaire à celle du premier article. Troisième et quatrième articles antennaires plus étroits que l'apex du second et ensemble nettement plus courts que ce dernier, uniformément brun foncé. Front obliquement strié, les stries brunes sur fond jaune créant un dessin complexe. Vertex dépourvu de carène postérieure ou de véritable sillon médian, sa surface subdivisée en trois zones sub-triangulaires, deux bordant les yeux, jaunes, chacune avec une large tache ovale rouge-brun au milieu et une troisième bordant la marge postérieure de la tête, rouge-brun avec une petite tache jaune. Rostre jaune brunâtre, atteignant au moins la moitié de l'abdomen.

Pronotum campanuliforme (fig. 1), jaune, taché de brun-rouge, portant de très courtes soies blanches couchées. Anneau étroit, finement strié transversalement, jaune brunâtre avec deux taches brunes submédianes et les bords bruns. Callosités grandes, occupant près de la moitié de la surface pronotale, mais peu marquées, quasi circulaires, totalement séparées l'une de l'autre et des marges latérales du pronotum, jaunes, portant de relativement larges taches arrondies brun-rouge. Bords latéraux du pronotum brun foncé, les marges finement carénées, flaves. Disque pronotal finement strié, les stries incurvées, brun à brun rougeâtre, portant cinq bandes claires longitudinales à faiblement obliques. Angles huméraux en pointes émoussées. Marge postérieure du pronotum quasi droite à la limite du mésoscutum, faiblement arrondie concave à hauteur des angles huméraux.

Mésoscutum découvert, portant deux larges fossettes latérales, sa zone centrale brune bordée de jaune.

Scutellum brun sauf l'apex jaune, portant de courtes soies blanches semi-dressées.

Hémélytres (fig. 1) jaunes tachés de brun, les taches brunes plus moins nettement confluentes et de largeur variable selon les zones. Clavus, corie et cunéus bien délimités. Exocorie largement brune, sauf une large tache jaune sub-basale. Fracture cunéale peu marquée, semblant limitée à un fin sillon blanc. Cunéus large, jaune basalement et apicalement, brun-rouge au milieu. Membrane normalement développée, brungris avec de larges nervures brunes à blanchâtres, l'apex de la grande cellule atteignant l'apex du cunéus.

Abdomen rouge-brun porteur de longues soies blanches semi-dressées.

Génitalias. Paratype mâle de l'ISNB. Paramère gauche (fig. 9) falciforme, son apophyse primaire en large crochet. Paramère droit (fig. 10) particulièrement long et large, le corps translucide sauf sur sa marge externe, l'apophyse primaire allongée, légèrement crochetée à l'apex. Phallus (fig. 11) long et grèle, tout comme le ductus seminis (Ds). Gonopore secondaire (G2) étroit. Thèque avec une marge nettement renforcée. Apex phallique pourvu d'un spicule (Sp).
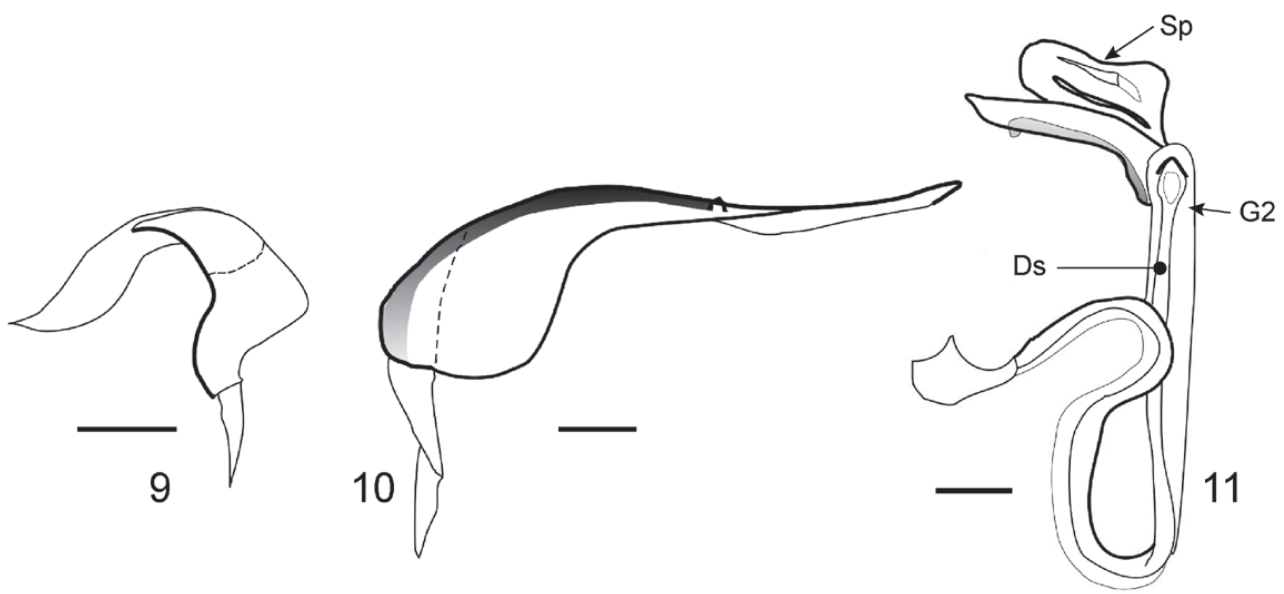

Fig. 9-11. - Xenocylapoides gorczycai n. gen., n. sp., génitalias du mâle paratype de l'ISNB. - 9, Paramère gauche en vue dorsale. - 10, Paramère droit en vue dorsale. - 11, Phallus (Ds : ductus seminis; G2 : gonopore secondaire; Sp : spicule). Échelles : 0,1 mm. 
Femelle (fig. 2-7). Globalement similaire au mâle, mais avec des hémélytres réduits ${ }^{2}$ à bords plus nettement arrondis, le pronotum plus nettement campanuliforme, les séparations clavus-corie et endoexocorie peu marquées, le cunéus absent, la membrane extrêmement réduite, formant un arc de cercle. Profémurs larges, épais, leur plus grande largeur juste après la base, jaune rougeâtre, porteurs de quelques soies semi-dressées. Tibias brunâtres porteurs de fines épines et de soies semi-dressées. Tarses jaunâtres, porteurs de soies blanches semi-dressées, apparemment constitués de trois articles.

Génitalias inconnus, les femelles à disposition n'étant pas suffisamment matures pour une analyse de ceux-ci.

Derivatio nominis. - La nouvelle espèce est dédiée à notre collègue Jacek Gorczyca (Silesian University, Bankowa, Pologne) pour ses importantes contributions à la connaissance des Cylapinae de l'Ancien Monde.

Habitat. - Collectée à Curicó, VII Región chilienne, Xenocylapoides gorczycai n. sp. appartient à la région biogéographique Andina, subrégion Chilena Central, province de Santiago (Morrone, 2001). Elle a été trouvée sur la canopée de la forêt subantarctique, sur le "Coihue" Nothofagus dombeyi Mirb. (Oerst.), l'une des plus hautes espèces du genre. Nous ne disposons d'aucune autre information sur sa biologie.

\section{AUTEURS CITÉS}

Carpintero D. L., 1998. - Miridae (p. 144-150). In : Morrone J. J. \& Coscarón S. (éds), Biodiversidad de Arthrópodos Argentinos. Una Perspectiva Biotaxonómica. La Plata : Ed. Sur, 599 p.

— 2009. - Insecta: Hemiptera: Heteroptera. In : Athor J. (éd.), Parque Costero del Sur: Naturaleza, conservación y patrimonio cultural. Buenos Aires : Fundación de Historia Natural "Félix de Azara", 528 p.

Carpintero D. L. \& Carvalho J. C. M., 1993. - An annotated list of the Miridae of the Argentine Republic (Hemiptera). Revista Brasileira de Biologia, 53 (3) : 397-420.

Carpintero D. L., Dellapé P. M. \& Melo M. C., 2006. - New Records of Heteroptera (Hemiptera) from Argentina. Zootaxa, 1129 : 1-22.

Carvalho J. C. M., 1957. - Catálogo dos Mirídeos do Mundo. Parte I. Subfamilias Cylapinae, Deraeocorinae, Bryocorinae. Arquivos do Museu Nacional, 44 : 1-158.

— 1976. - Mirídeos Neotropicais, CCII: um novo gênero e cinco espécies novas da coleção da Academia de Ciências da Califórnia (Hemiptera). Revista Brasileira de Biologia, 36 (3) : 715-721.

CARvalho J. C. M. \& Afonso C. R. S., 1977. - Mirídeos Neotropicais, CCVIII: Sobre uma coleção enviada para estudio pela academia de ciências da California. Revista Brasileira de Biologia, 37 (1) : 7-16.

Carvalho J. C. M. \& Carpintero, D. L., 1985. - Mirídeos Neotropicais, CCLXVIII: Dois Gêneros Novos da República Argentina. Anais da Academia Brasileira de Ciencias, 57 (4) : 507-511.

Carvalho J. C. M. \& Costa L. L. A., 1994. - The genus Fulvius from the Americas (Hemiptera: Miridae). Anales de Instituto de Biología de la Universidad Nacional Autónoma de México, serie Zoología, 65 (1) : 63-135.

Carvalho J. C. M. \& Ferreira P. S. F., 1994. - Mirideos neotropicais, CCCLXXXVIII: chave para os generos de Cylapinae Kirkaldy, 1903 (Hemiptera). Revista Ceres, 41 (235) : 327-334.

ChÉrot F., CARPINTERo D. \& WOLSKI A., (soumis). - New record and redescription of the monotypic genus Comefulvius Carvalho \& Carpintero, 1985 (Hemiptera: Heteroptera: Miridae: Cylapinae). Zootaxa.

Dellapé P. M., Melo M. C. \& Carpintero D. L., 2010. - New records of Dipsocoromorpha, Cimicomorpha and Pentatomomorpha (Hemiptera: Heteroptera) from Argentina. Zootaxa, 2436 : 57-64.

GorczYCA J., 1997. - Xenocylapidius tamasi gen. et sp. n. from New Caledonia (Heteroptera: Miridae: Cylapinae). Polish Journal of Entomology, 66 : 177-184.

2000. - A systematic study on Cylapinae with a revision of Afrotropical Region (Heteroptera, Miridae). Bankowa : Wydawnictwo Universytetu Śląskiego, 176 p.

2006. - The catalogue of the subfamily Cylapinae Kirkaldy, 1903 of the World (Hemiptera, Heteroptera, Miridae). Monographs of the Upper Silesian Museum, 5 : 1-100.

\footnotetext{
${ }^{2}$ Les hémélytres étant réduits mais recouvrant les tergites 6 et 7, ce cas de réduction alaire pourrait apparemment être classé dans la catégorie submacroptère au sens de SCHUH \& SLATER (1995).
} 
Melo M. C., Dellapé P. M., Carpintero D. L. \& Coscarón M. C., 2004. - Inventario de Lygaeoidea, Miridae y Reduviidae (Heteroptera) en los esteros de Iberá, Corrientes. Revista de la Sociedad Entomológica Argentina, 63 (1-2) : 59-69.

Melo M. C., Dellapé P. M., Carpintero D. L. \& Montemayor S. I., 2011. - Heteroptera (Hemiptera) from the Chaco National Park. Zootaxa, 2999 : 1-19.

Morrone, J. J., 2001. - Biogeografía de América Latina y el Caribe. Manuales \& Tesis Sociedad Entomológica Aragonesa, 3 : 1-150.

NAnNi A. S., Magnano A. L. \& CARpintero D. L., 2011. - Los Heteroptera (Insecta Hemiptera) edáficos del INTA Delta del Paraná (partido de Campana, Buenos Aires). Variación espacial y temporal del elenco de las especies en distintos usos de la tierra. Revista del Museo Argentino de Ciencias Naturales, (N. S.) 13 (1) : 27-39.

Prado C. E., 2008. - Conocimiento actual de Hemiptera-Heteroptera de Chile con lista de especies. Boletín del Museo Nacional de Historia Natural, 57 : 31-75.

Schun R. T. \& Slater J. A., 1995. - True Bugs of the World (Hemiptera, Heteroptera). Classification and Natural History. Ithaca \& London : Cornell University Press, XII $+337 \mathrm{p}$.

Wolski A. \& Henry T. J., 2012. - Revision of the New World species of Peritropis Uhler (Heteroptera: Miridae: Cylapinae). Insect Systematics \& Evolution, 43 : 213-270.

\section{La Société entomologique de France présente au $17^{\mathrm{e}}$ Colloque de Biologie de l'Insecte}

La Société entomologique de France était partenaire du $17^{e}$ Colloque de Biologie de l'Insecte, qui s'est tenu à Montpellier du 7 au 9 novembre 2013 (120 participants).

Yvan Rahbé, chercheur à l'INRA et membre de la SEF, a présenté notre Société sous forme d'un diaporama, rappelant ses différentes activités (sorties entomologiques, organisation d'un colloque en 2013, publications des membres, ses trois journaux entomologiques, ...). En fin de colloque, deux doctorantes ayant fait une présentation ont été retenues pour recevoir les deux bourses 2013 de la SEF d'un montant de $400 €$. Il s'agit de :

Karen MulLeR, doctorante à Dijon (sous la direction de Jérôme Moreau), UMR 6282 Géosciences, Université de Bourgogne, 6 boulevard Gabriel, F - 21000 Dijon $<$ Karen.Muller @u-bourgogne.fr>. Titre de la communication : "Influence du cépage de vigne sur le succès reproducteur des mâles chez l'Eudémis (Lobesia botrana) et conséquences sur le potentiel reproductif des femelles" (en collaboration avec D. Thiery, Y. Morret, J. Moreau).

Pascaline Dumas, doctorante à Montpellier (sous la direction d'Emmanuelle d'Alençon et Gaël Kergoat (INRA), MUM2-UMR DGIMI Université, place Eugène-Bataillon, F - 34095 Montpellier <Pascaline.Dumas@supagro.inra.fr>. Titre de la communication : "Questioning the status of the two "fall army worm" (Spodoptera frugiperda, Noctuidae) host races using integrative approaches" (en collaboration avec A.-L. Clamens, S. Gimenez, G. Kergoat, E. d'Alençon). 